\title{
SÍNTESE E CARACTERIZAÇÃO DE ÓXIDO DE GRAFENO PARA USO EM CAPACITOR ELETROQUÍMICO
}

\author{
SYNTHESIS AND CHARACTERIZATION OF GRAPHENE OXIDE FOR USE IN \\ ELECTROCHEMICAL CAPACITOR
}

\author{
Erica Cristina Almeida ${ }^{1}$, \\ Renan Neres Santos', \\ Elilton Rodrigues Edwards ${ }^{3}$
}

RESUMO: Grafeno é uma monocamada com estrutura hexagonal de átomos de carbono e que tem se tornado um material de grande interesse para os pesquisadores da nanotecnologia e nanociência. Neste artigo foi analisada a viabilidade da aplicação do grafeno tanto oxidado como reduzido para uso como eletrodo em capacitores eletroquímicos. A síntese do óxido de grafeno foi obtida através do método modificado de Hummels-Hofmann e posteriormente a redução química e térmica deste material. As caracterizações dos materiais foram realizadas pelas técnicas de Difração de raios-x (DRX), Espectroscopia de Infravermelho e Espectroscopia Raman. Análises por DRX apresentou uma esfoliação suave para o óxido de grafeno tendo como precursora a grafite. $O$ resultado da espectroscopia de infravermelho apresentou grupos oxigenados que foram inseridos no processo da oxidação, corroborado pela técnica de espectroscopia Raman. O grafeno sintetizado foi analisado por espectroscopia Raman e apresentou grafeno de multifolhas. Os eletrodos obtidos foram caracterizados pela técnica de Voltametria Cíclica e os voltamogramas mostraram que o óxido de grafeno apresentou melhores resultados para aplicação como capacitor eletroquímico, comparado com o grafeno reduzido, evidenciando que a incorporação de grupos oxigenados à superfície do óxido melhorou a formação da dupla camada elétrica.

Palavras-chave: Grafite. Redução eletroquímica. Óxido de grafeno. Grafeno reduzido.

\footnotetext{
' Graduação em Química pela Universidade de São Paulo (1999), Mestrado em Química pela Universidade de São Paulo (20or) e Doutorado em Engenharia e Tecnologias Espaciais pelo INPE/LAS (2006). Professora Adjunta na Universidade Estadual de Santa Cruz no curso de Engenharia Mecânica. Tem experiência na área Química com ênfase em eletroquímica do estado sólido e na área de Engenharia de Materiais e Metalúrgica, com ênfase em materiais carbonosos, atuando principalmente nos seguintes temas, compósitos poliméricos, impressão $3 \mathrm{D}$ e Capacitores eletroquímicos. E-mail: ecalmeida@uesc.br.

${ }_{2}^{2}$ Possui licenciatura em Química pela Universidade Estadual do Sudoeste da Bahia - UESB - campus Jequié (2013), Especialização em Ensino de Química pela Faculdade do Vale Elvira Dayrell-FAVED (2019), Mestrado Acadêmico em Ciência, Inovação e Modelagem em Materiais pela Universidade Estadual de Santa Cruz - UESC (20r6). Atualmente é professor da educação básica (ensino médio-integral) na Secretaria de Educação do Estado da Bahia - Sec/Ba . E-mail: neresquimica@yahoo.com.br.

${ }^{3}$ Possui graduação em Engenharia Industrial Química pela EEL/USP-Lorena (200o), Mestrado em Engenharia de Materiais pela mesma instituição (2003), Doutorado em Engenharia e Tecnologias Espaciais pelo INPE/LAS (2008) e pós-doutorado na UNESP/Guaratinguetá (2013). Atualmente é Professor Titular na Universidade Estadual de Santa Cruz - UESC, pesquisador na área de materiais para aplicações aeronáuticas com ênfase na fabricação de nanotubos de carbono, purificação, dispersão e funcionalização para fabricação de compósitos nanoestruturados; síntese de compósitos nanoestruturados; física e química de superfícies voltadas para fabricação de novos materiais com aplicações tecnológicas. Também desenvolve pesquisa na área de modelagem computacional na área de materiais. E-mail: eredwards@uesc.br.
} 
ABSTRACT: Graphene is a monolayer with a hexagonal structure of carbon atoms and has become a material of great interest to researchers in nanotechnology and nanoscience. In this paper the viability of the application of both oxidized and reduced graphene for use as electrodes in electrochemical capacitors was analyzed. The synthesis of graphene oxide was obtained through the modified method of Hummers-Hoffmann and later the chemical and thermal reduction of this material. The materials were characterized by X-ray Diffraction (XRD), Infrared Spectroscopy and Raman Spectroscopy. Analysis by XRD showed a little exfoliation for the graphene oxide with as precursor the graphite. The results of infrared spectroscopy showed oxygen groups that were inserted in the oxidation process, corroborated by the Raman spectroscopy technique. Synthesized graphene was analyzed by Raman spectroscopy and showed multi-sheet graphene. The electrodes obtained were characterized by the cyclic voltammetry technique and the voltammograms showed that graphene oxides presented better results as an electrochemical capacitor, compared to the reduced graphene, evidencing that the incorporation of oxygen groups on the surface of the oxide improved the formation of the layer electric double.

Keywords: Graphite. Electrochemical reduction. Graphene oxide. Reduced graphene.

\section{INTRODUÇÃO}

Os materiais nanoestruturados de carbono apresentam estruturas cuja escala de comprimento é da ordem de uns poucos nanômetros, tipicamente entre I a loo nanômetros, sendo integrados a esse grupo os nanotubos de carbono e os fulerenos cuja descoberta foi atribuída a Iijima (IIJIMA,199I). O grafeno foi descoberto no final de 2004 no centro de Nanotecnologia da Universidade de Manchester no Reino Unido, pelos cientistas russos e prêmio Nobel Andre Geim e Konstantin Novoselov (GEIM; NOVOSELOV, 20Io). O grafeno apresenta uma monocamada com estrutura hexagonal de átomos de carbono o que o torna um material de grande interesse para os pesquisadores da nanotecnologia e nanociência $(\mathrm{N} \& \mathrm{~N})$. O principal interesse na pesquisa com este novo material se deve às suas excelentes propriedades, principalmente como condutor elétrico que é similar ao cobre e como condutor de calor que supera todos os outros materiais conhecidos. É um material mais forte e mais duro do que o diamante e pode ser esticado em $25 \%$ do seu comprimento (PEI; CHENG, 2012). Chen (CHEN et al., 2010) verificou um valor de mobilidade intrínseca, que representa a velocidade com a qual o material conduz uma corrente elétrica igual a 200.000 $\mathrm{cm}^{2} /$ V.s, sendo superior àquela verificada para os nanotubos de carbono, sendo considerada a maior mobilidade já apresentada por qualquer sólido.

Este material nanoestruturado apresenta características excepcionais, inclusive para armazenamento de energia, pois apresenta uma alta área superficial, boa mobilidade a 
baixas temperaturas, inclusive em temperatura ambiente, em comparação ao silício. E devido a estas excelentes propriedades tornou-se uma ótima alternativa para aplicações em circuitos integrados, como chip, condutor LCD, touchscreen, células solares, transistores, fotodetectores e ultra-capacitores (GIRALDO, 20II). O principal objetivo deste trabalho é o estudo da viabilidade da aplicação do óxido de grafeno, tanto na forma reduzida quanto na forma oxidada, para utilização na construção de eletrodos capacitores eletroquímicos para armazenamento de energia. Este trabalho tem fundamental importância nos dias atuais em que vários grupos de pesquisa, em todo mundo, buscam formas alternativas para geração de energia limpa, haja vista a visível escassez de energia em um futuro não tão distante do atual. As literaturas apresentam muitos métodos para produção do óxido de grafeno reduzido (RGO) (LIU, 2013), incluindo a clivagem micromecânica (NOVOSELOV; GEIM, 2004), deposição química na fase vapor (KIM et al., 2009), rápido tratamento térmico (ZHU et al., 20I0) e redução química do óxido de grafeno (GO) (STANKOVICH et al., 2007; LIAO; MITTAL, 20II). Dentre estes métodos a redução química do óxido de grafeno é considerado o método mais eficiente ( $\mathrm{LOH}$ et al., 2010).

Neste trabalho a síntese e redução do óxido de grafeno foram obtidas pelo método modificado de Hummers-Offerman e posteriormente foi feita a redução química e térmica no material. As técnicas utilizadas para a caracterização estrutural foram a Difração de raios-X (DRX), Espectroscopia de Infravermelho (IR) e Espectroscopia Raman. Os eletrodos obtidos foram caracterizados pela técnica de Voltametria Cíclica (VC).

\section{2 - MATERIAIS E MÉTODOS}

O óxido de grafite (GO) foi produzido a partir da redução da grafite natural (fornecida pela Nacional Grafite) pelo método de Hummers modificado (HUMMERS et al.,I957). Em um béquer contendo $2 \mathrm{~g}$ de grafite e sob agitação em banho de gelo foram adicionados I $\mathrm{g}$ de nitrato de sódio $\left(\mathrm{NaNO}_{3}\right)$ e $46 \mathrm{~mL}$ de ácido sulfúrico concentrado $\left(\mathrm{H}_{2} \mathrm{SO}_{4}\right)$. Após 5 minutos em agitação, foi adicionado $6 \mathrm{~g}$ de permanganato de potássio $\left(\mathrm{KMnO}_{4}\right)$ lentamente e com controle de temperatura para não exceder a temperatura de $20{ }^{\circ} \mathrm{C}$. O banho de gelo foi retirado após 5 minutos e o sistema foi aquecido a $35^{\circ} \mathrm{C}$ por 30 minutos quando então foi observada a formação de uma pasta de coloração marrom. Em seguida, $92 \mathrm{~mL}$ de água foram lentamente adicionados ao sistema que estava mantido em agitação magnética. Após is 
minutos foram adicionando $80 \mathrm{ml}$ de água na temperatura de $60{ }^{\circ} \mathrm{C}$ e $20 \mathrm{~mL}$ de peróxido de hidrogênio $\left(\mathrm{H}_{2} \mathrm{O}_{2}\right)$ a 30\% a fim de reduzir o permanganato residual e o dióxido de manganês, a mistura adquiriu uma coloração amarela. Por fim, o sistema foi filtrado e o resíduo lavado a quente e foi obtido o óxido de grafite. $\mathrm{O}$ pó obtido foi disperso com água em um becker no banho de ultrassom. Em seguida foi filtrado e secado em estufa a $60{ }^{\circ} \mathrm{C}$ por $2 \mathrm{~h}$, então foi obtido o óxido de grafeno (GO).

\section{I REDUÇÕES TÉRMICAS DO GO (RGO)}

Foram adicionados $20 \mathrm{mg}$ de óxido e grafite em $6 \mathrm{~mL}$ de água destilada e levemente disperso em banho de ultrassom por um período de 15 minutos. Em seguida, $30 \mathrm{~mL}$ de $\mathrm{N}, \mathrm{N}$ dimetilacetamida (DMAc) foi adicionada na solução que voltou a ser sonicado por mais 5 minutos. Em seguida foram preparados $36 \mathrm{ml}$ de óxido de grafite em suspensão com uma mistura de DMAc/ $\mathrm{H}_{2} \mathrm{O}(0,56 \mathrm{mg} / \mathrm{mL})$. O material foi colocado dentro de um forno microondas por um período de 2 minutos. Em seguida a suspensão foi filtrada a vácuo e posteriormente lavado com $30 \mathrm{~mL}$ de etanol por três vezes. Em seguida, o material foi transferido para um béquer, contendo $15 \mathrm{ml}$ de água e novamente tratado com ultrassom e seco em estufa por $\mathrm{ih}$.

\subsection{EQUIPAMENTOS UTILIZADOS}

Foram utilizados ultrassom (UltraSonic Cleaner-UNIQUE), Centrífuga EBA 20 (Hettich Zentrifugen), Microondas doméstico (Philco de I200 W), espectrofotômetro Thermo Scientific Nicolet, modelo isıo SMART OMNI - Transmission com uma faixa espectral de 4000 a $400 \mathrm{~cm}^{-1}$, difratômetro Rigaku modelo miniflex 6oo, com radiação $\mathrm{Cu} \mathrm{K \alpha}$ e $2 \theta=5-80^{\circ}$ com velocidade de varredura de $20 \%$ min a $40 \mathrm{Kv}$ e $15 \mathrm{~mA}$, equipamento Micro Raman Sistema 2000 da Renishaw, no Laboratório Associado de Materiais do Instituto Nacional de Pesquisas Espaciais. A energia de excitação do laser de Ar foi de 2,4I eV com comprimento de onda de $514,5 \mathrm{~nm}$, fita de $\mathrm{pH}$.

\section{MATERIAIS E MÉTODOS}

O processo de oxidação insere grupos funcionais no espaçamento entre as camadas de nano lâminas de folhas de grafite podendo ocasionar a diminuição do tamanho médio dos cristais de grafite. Isto ocorre porque a inserção de grupo contendo oxigênio (grupos epóxi, hidroxil e 
carbonil) diminui a força de Van der Waals entre as intercamadas propiciando a separação destas folhas (YUE et al., 20II) e posterior diminuição do tamanho médio dos cristais.

A Figura I mostra os resultados de Infravermelhos (IV) da grafite como material de partida, para comparação, e do óxido de grafeno. O espectro de IV da grafite (Figura I A) apresenta uma banda em torno de $2200 \mathrm{~cm}^{-1}$ e está relacionada à presença de impurezas. Após o processo de oxidação fica evidente no espectro IV do GO (Figura I B) a introdução de novos grupos funcionais, confirmando assim o processo de oxidação. Observou-se a presença de bandas nas regiões de 3600-3000 $\mathrm{cm}^{-1}$,características de grupos oxigenados como hidroxila $(\mathrm{OH})$, na região de $1850-1610 \mathrm{~cm}^{-1}$ de grupos carbonila $(\mathrm{C}=\mathrm{O})$ e na região de $1300-1050 \mathrm{~cm}^{-1}(\mathrm{C}-\mathrm{O})$ possível grupos epóxi.
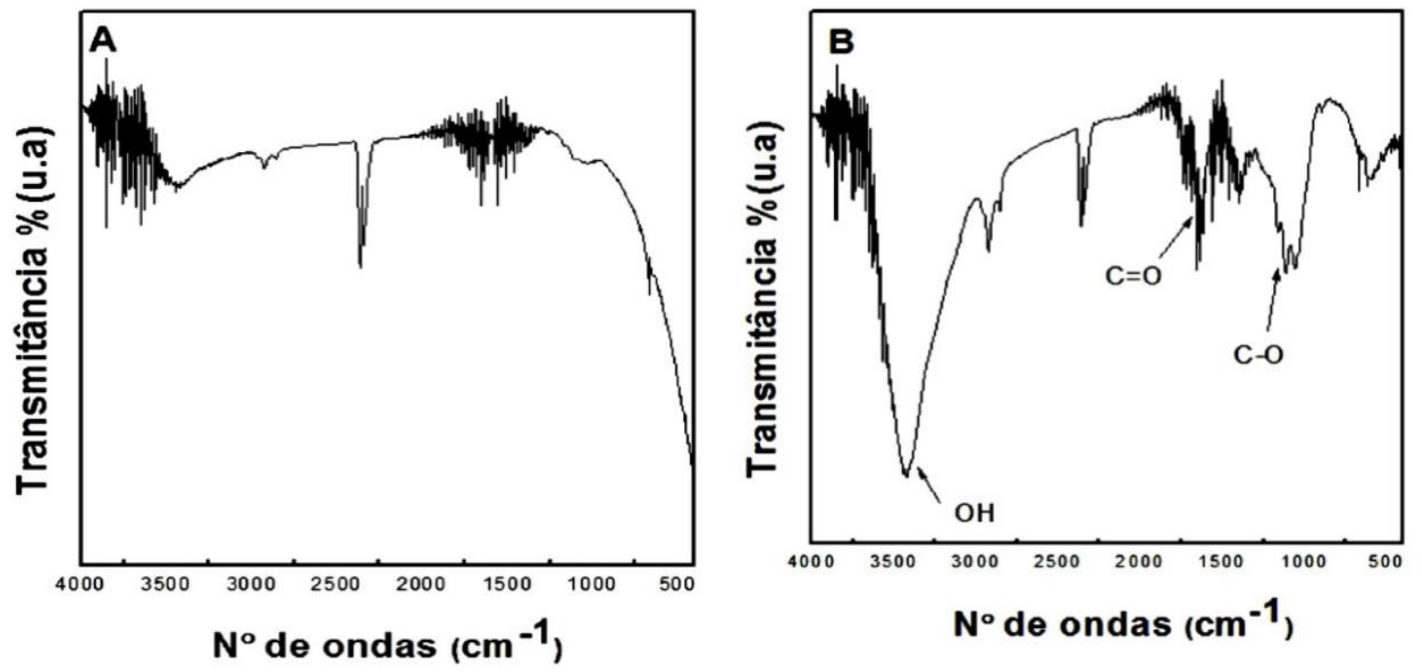

Figura I: Espectros de infravermelho da grafite pura (A) e do óxido de grafeno (B) obtido através do processo de oxidação.

A Figura 2 mostra os padrões de difração da grafite e do óxido de grafeno. A partir da Figura 2(A) foi observado que os padrões de difração da grafite apresentou picos intensos e estreitos, um padrão característico da refração (0oz) e um pequeno pico de baixa intensidade devido a refração (004). A figura 2(B) mostra que o padrão de difração do GO não apresentou picos, mais bandas. Isto ocorre porque o espaçamento na rede cristalina do óxido de grafeno não é bem definida como ocorre na grafite altamente ordenada. 

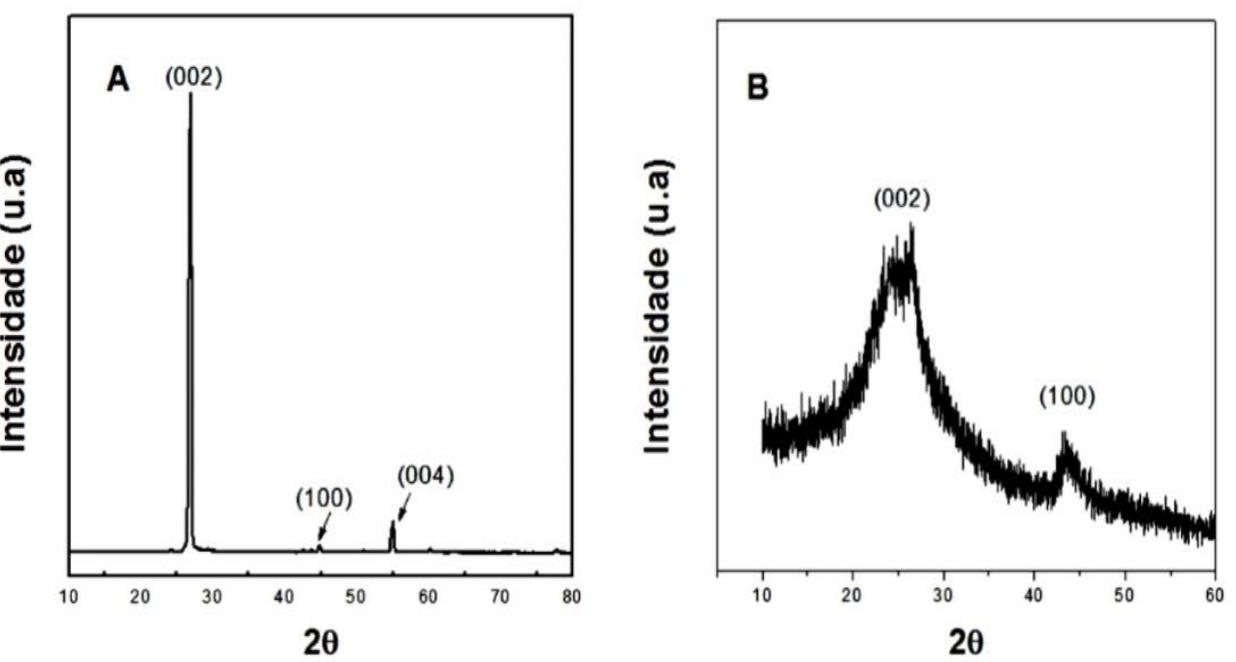

Figura 2: Padrões de Difração de Raios- $\mathrm{X}$ da grafite (A) e do seu óxido de grafeno (B).

A Figura 3 mostra o espectro normalizado de espalhamento Raman de primeira e segunda ordem da grafite e do óxido de grafeno. A partir da Figura 3 (A) o espectro da grafite apresenta, na banda de primeira ordem, duas bandas dominantes chamadas de banda D (desordem) e $\mathrm{G}$ (grafitica). A banda D encontra-se em aproximadamente em $136 \mathrm{~cm}^{-1}$, sendo apresentada como uma banda de desordem na estrutura cristalina, o que não ocorre em cristais perfeitos. A máxima intensidade da banda $G$ ocorre em aproximadamente $1585 \mathrm{~cm}^{-1}$ sendo uma característica da estrutura grafítica ordenada. Comparando-se os espectros do óxido de grafeno (Figura 3 B) com os da grafite (Figura 3 A) foi observado um aumento da intensidade da banda $\mathrm{D}$, esse aumento é um indicativo de muitos defeitos na estrutura cristalina decorrente dos processos de oxidação.

A intensidade elevada da banda $\mathrm{D}$, na Figura $3 \mathrm{~B}$, está relacionada aos defeitos na estrutura cristalina devido à desordem causada pela inserção de grupos funcionais como oxigênio formando carbonos hibridizados que favoreceu a desordem na estrutura cristalina de carbono da grafite para formação do óxido de grafite. Desta forma, esta banda também pode ser associada ao grau de oxidação da grafite durante o processo de oxidação. A banda $G$ está associada à ordenação da estrutura grafitica em que se verifica o tamanho médio dos agregados ou as regiões de hibridização $\mathrm{sp}^{2}$ relacionados com a banda D (TUINSTRA; KOENIG, I970) conforme a equação I. 


$$
L_{a}=\frac{I_{D}}{I_{G}}
$$

Sendo $\mathrm{L}_{\mathrm{a}}$ o tamanho médio dos agregados $\mathrm{sp}^{2}$ e $\mathrm{I}_{\mathrm{D}}$ e $\mathrm{I}_{\mathrm{G}}$ as intensidades destas bandas. Esta relação permite controlar as propriedades eletrônicas do óxido de grafeno pela diminuição do tamanho dos grãos, podendo passar de isolante a semicondutor e a metal, o que não é possível no óxido de grafeno. O espectro de Raman do óxido de grafeno apresenta predominância da banda $\mathrm{D}$ e $\mathrm{G}$ (JIN et al., 2013) e que a banda $\mathrm{D}$, em torno de $1340 \mathrm{~cm}^{-1}$ aparece no espectro do GO devido à introdução de grupos oxigenados na rede cristalina o que confirma o processo de oxidação. Os resultados das análises do espectro de Raman do óxido de grafeno apresentaram uma maior predominância da banda $\mathrm{D}$ e $\mathrm{G}$ no espectro do $\mathrm{GO}$ devido à introdução de grupos oxigenados na rede cristalina (RUMMELI et al., 2010) o que confirmou o processo oxidativo. Resultados semelhantes foram encontrados por (CHEN et al., 2010; LIU et al., 2013) para o óxido de grafeno em que foi observada uma maior intensidade da banda $G$, no espectro Raman, sugerindo que o material analisado realmente trata-se do óxido de grafeno.

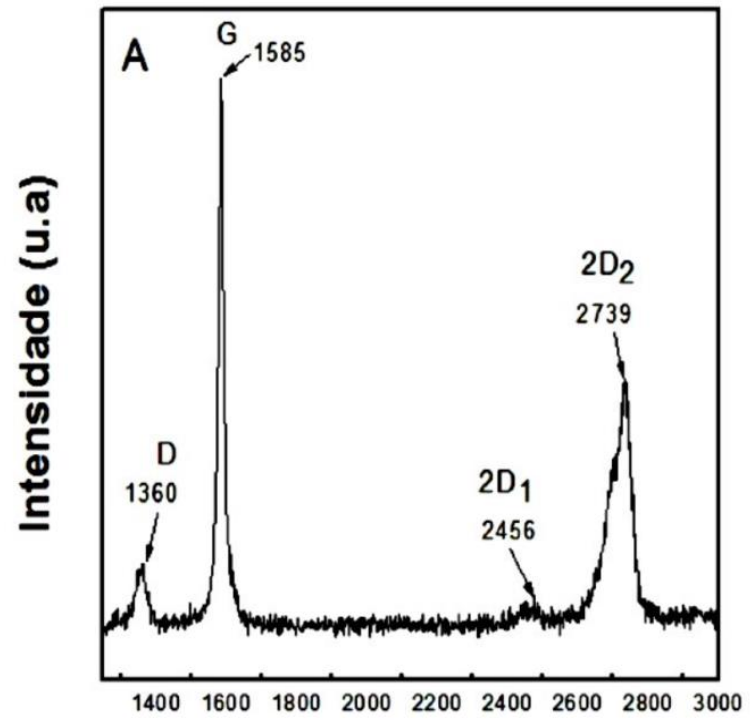

Deslocamento Raman (cm-1)

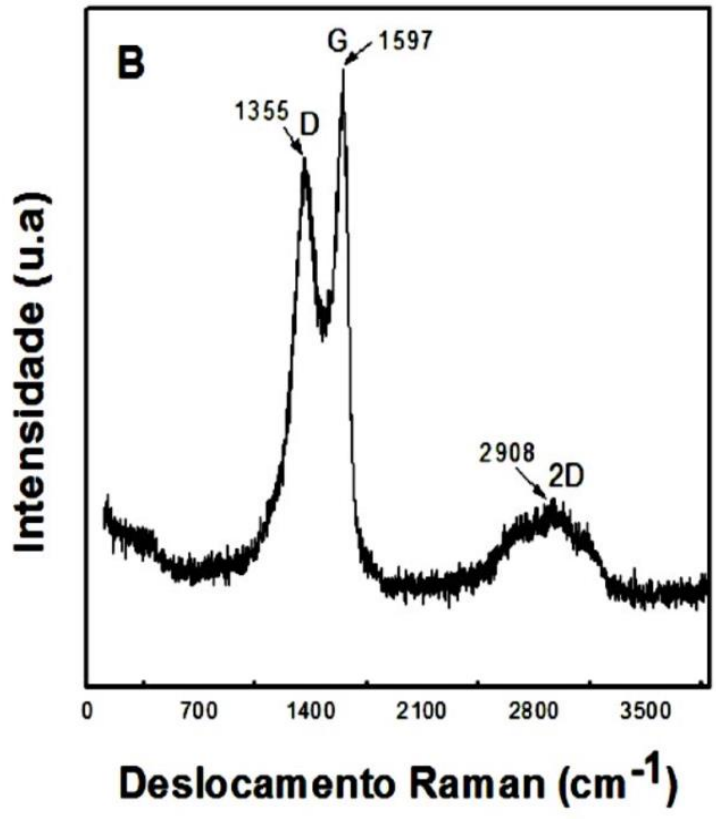

Figura 3: Espectros de espalhamento Raman de primeira e segunda ordem da grafite (A) e do óxido de grafeno (B). 


\section{I CARACTERIZAÇÕES DO GRAFENO REDUZIDO (RGO).}

A Figura 4 mostra o espectro de espalhamento Raman de primeira e segunda ordem da grafite e do óxido de grafeno reduzido. A partir desta figura foi observado que na redução do óxido de grafeno ocorreu a diminuição significativa destas bandas e não seu desaparecimento completo. Situação semelhante foi encontrada por Lui (LIU et al., 2013) e significa que, conforme já citado neste trabalho, a redução deixou resíduos dos grupos funcionais oxigenados. As bandas $\mathrm{D}$ que surgem no óxido de grafeno reduzido referem-se à desordem estrutural com formações de defeitos de contornos na grafeno e confirmado em (LIU et al., 2013). As bandas $2 \mathrm{D}$ estão relacionadas às camadas presentes no grafeno e permanece em ambos espectros sendo um indicativo de que se trata de grafeno.

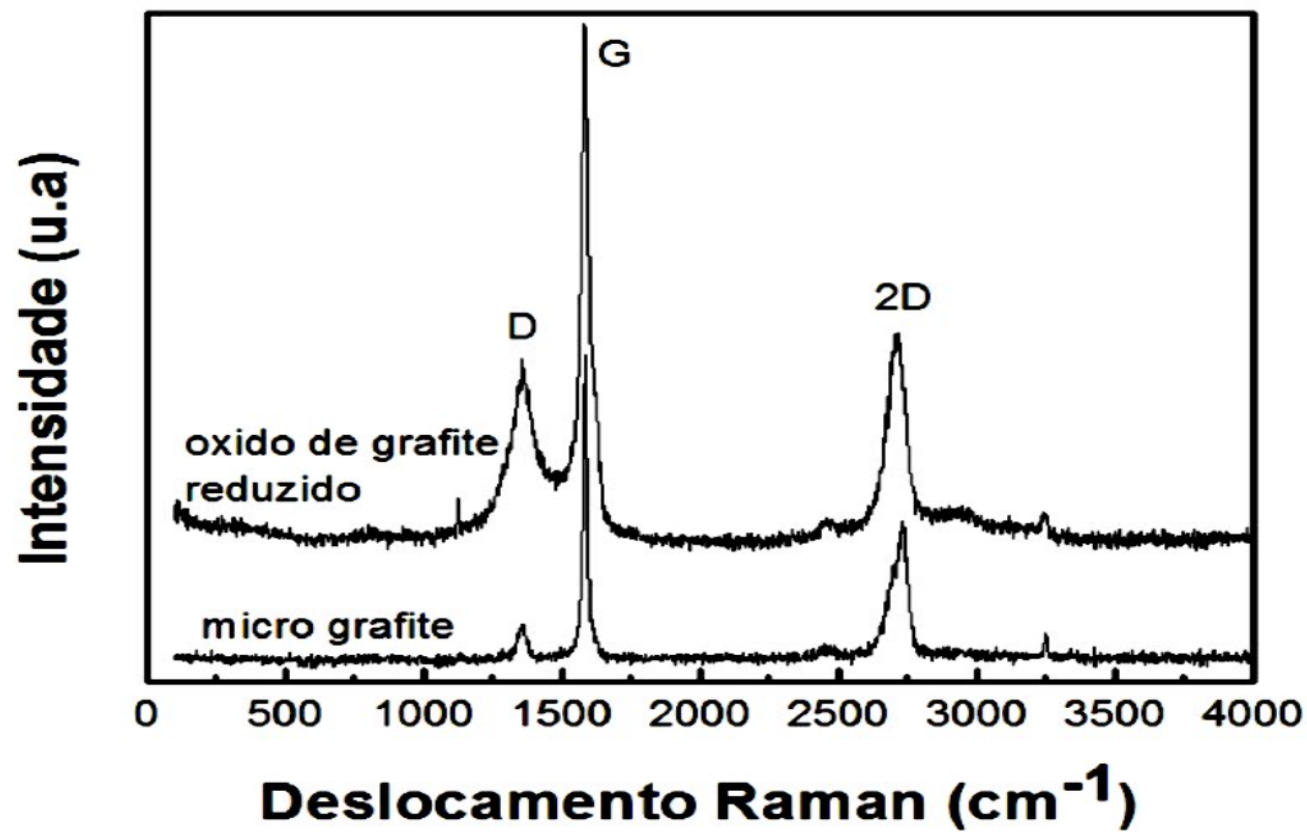

Figura 4: Espectros de espalhamento Raman de primeira e segunda ordem da grafite e do óxido de grafeno reduzido.

A Figura 5 mostra o espectro de espalhamento Raman de segunda ordem da grafite e do óxido de grafeno reduzido. A partir desta figura foi observado que as bandas $\mathrm{G}^{\prime}$ ou $2 \mathrm{D}$ do grafite e do óxido de grafeno reduzido, têm estruturas diferente uma vez a grafite apresenta uma divisão da banda em $2 \mathrm{D}_{1}$ e $2 \mathrm{D}_{2}$ enquanto que não é observado no óxido de grafeno 
Revista Ibero- Americana de Humanidades, Ciências e Educação- REASE open 2 Access

reduzido, logo é possível dizer que a redução ocorreu efetivamente, pois é a banda G' ou $2 \mathrm{D}$, a banda utilizada para a identificação do grafeno. Essa característica nas bandas de ambos é apresentada na literatura como confirmação de que o material reduzido trata-se do grafeno (RUMMELI et al., 2010). Os resultados da análise de Raman do grafeno sintetizado neste trabalho foram comparados com os dados na literatura (FERRARI, 2007) e os resultados mostraram que não se trata de nanofolhas e sim de multi folhas de grafeno, uma vez que essa análise pode ser verificada através da relação de intensidade do pico $\mathrm{G}$ com a intensidade do pico 2D (FERRARI et al., 2010; WANG et al., 2008).
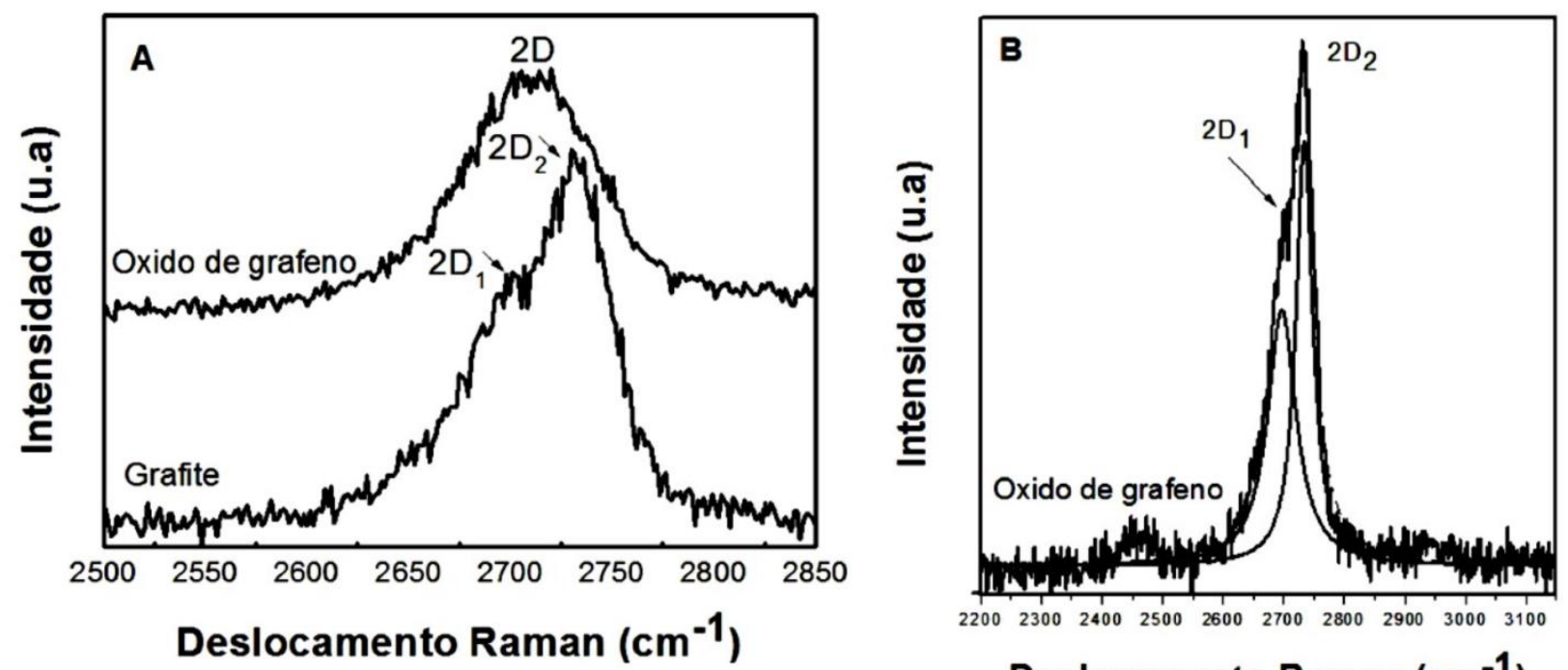

Figura 5: Espectros de espalhamento Raman de segunda ordem da grafite e do óxido de grafeno reduzido.

\subsection{VOLTAMETRIAS CÍCLICAS}

A Figura 6 mostra a voltamograma cíclico da grafite (6A), do óxido de grafeno (6B) e óxido de grafeno reduzido (6C) utilizando-se velocidade de varredura de $20 \mathrm{mV} . \mathrm{s}^{-1} \mathrm{e}$ electrólito o,5 mol. $\mathrm{L}^{-1}$ de $\mathrm{H}_{2} \mathrm{SO}_{4}$ em um intervalo de potencial de -0,2 a o,6 V vs. $\mathrm{Ag} / \mathrm{AgCl}$. $\mathrm{O}$ comportamento capacitivo de um eletrodo ideal é expresso na aparência de um voltamograma retangular. Neste tipo de armazenamento de energia o fenômeno é puramente eletrostático e a corrente é independente do potencial. Por outro lado, quando o eletrodo apresenta propriedades pseudo capacitivas, ou seja, características devido a processos redox reversíveis, pela transferência de carga faradaica, verifica-se no material do eletrodo um desvio deste 
comportamento (FRACKOWIAK; BÉGUIN, 200I). O eletrodo que apresentou um melhor comportamento eletroquímico foi o óxido de grafeno. A grafite apresentou um comportamento eletroquímico muito inferior tanto ao eletrodo GO como RGO. A explicação para o desempenho do eletrodo GO deve-se a maior presença de grupos funcionais. Esses grupos estão facilitando a transferência eletrônica na superfície do eletrodo. Os eletrodos de GO e RGO foram ciclados em diferentes velocidades de varredura e os resultados obtidos da densidade de corrente estão mostrados na Tabela I. A partir dos dados desta tabela foram construídos gráficos de densidade de corrente em função da velocidade de varredura cujo coeficiente angular da reta resultante deve ser igual à capacitância do eletrodo. Foram encontrados valores de capacitância de 209 F.m² para o óxido de grafeno (GO) e de 104 F.m ${ }^{-2}$ para o óxido de grafeno reduzido (RGO).

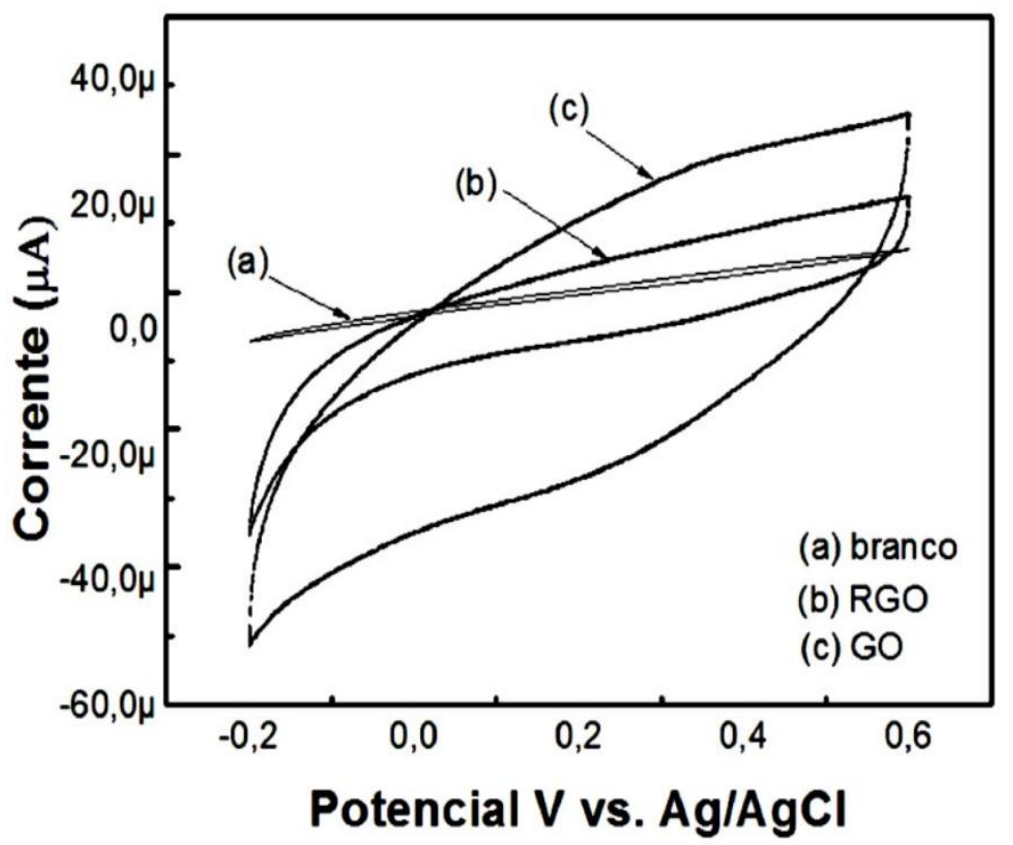

Figura 6: Voltamograma cíclico da grafite (A), do óxido de grafeno (B) e Óxido de grafeno Reduzido (C), utilizando velocidade de varredura de $20 \mathrm{mV} \cdot \mathrm{s}^{-1}$ e eletrólito $0,5 \mathrm{~mol} \cdot \mathrm{L}^{-1}$ de $\mathrm{H}_{2} \mathrm{SO}_{4}$ num intervalo de potencial de -0,2 a o,6 V vs. $\mathrm{Ag} / \mathrm{AgCl}$.

Tabela r. Densidades de correntes obtidas em o,2 V vs. $\mathrm{Ag} / \mathrm{AgCl}$ em diferentes velocidades de varredura (v) para os eletrodos de GO e RGO. 


\begin{tabular}{ccccccc}
\hline$v\left(\mathbf{m V} \cdot \mathbf{s}^{-1}\right)$ & $\mathbf{1 0 0}$ & $\mathbf{8 0}$ & $\mathbf{6 0}$ & $\mathbf{4 0}$ & $\mathbf{2 0}$ \\
\hline & GO & 22,6 & 19,2 & 15,5 & 11,3 & 5,6 \\
$\mathbf{j}(\mathbf{A}) \cdot \mathbf{m}^{-2}$ & RGO & 9,2 & 7,4 & 5,1 & 2,9 & 1,09
\end{tabular}

\section{${ }_{4}$ CONCLUSÕES}

Neste trabalho foram realizadas a oxidação da grafite seguida de sua esfoliação via centrifugação e posterior redução química para obtenção do óxido de grafeno, sendo esta a primeira parte do trabalho. $\mathrm{O}$ óxido de grafeno reduzido foi obtido na segunda parte através da oxidação química deste material. Os materiais obtidos foram analisados por espectroscopia de infravermelho, difração de Raios-x e espectroscopia Raman. Os resultados apresentaram importantes informações tais como a qualidade da esfoliação obtida no DRX. Os dados obtidos mostraram que ocorreu uma suave esfoliação no óxido de grafeno obtido da grafite comercial. A análise por espectroscopia de infravermelho apresentou a presença de grupos oxigenados que foram inseridos no processo de oxidação. Estes dados foram comparados com os da literatura e mostraram valores esperados, o que corroborou com os resultados obtidos pela técnica de Espectroscopia Raman. No processo de redução da grafite, segunda parte do trabalho, foi feita a análise de espectroscopia de infravermelho para identificação da incorporação de grupos funcionais após a redução. Foi observado que aqueles grupos funcionais inseridos durante a oxidação ainda estavam presentes após a redução do óxido. Isto se deve ao fato de que durante a redução, o material ainda está submetido à presença de grupos funcionais que reagem com a superfície do grafeno pelo ataque às ligações $s^{2}$, porém pelos resultados da espectroscopia de espalhamento Raman foi observado que os materiais reduzidos são multi folhas de grafeno. Amostras de GO e RGO foram caracterizadas por análise voltametria eletroquímicas a fim de verificar sua disponibilidade para possível utilização como capacitores. Os resultados de voltametria mostraram que os óxidos sintetizados apresentaram melhores condições de uso como capacitor do que os RGO. Isto se deve à grande incorporação de grupos oxigenados à superfície do $\mathrm{GO}$ em comparação ao RGO. O processo de redução do óxido retirou grupos oxigenados da superfície do GO e isto o tornou menos hidrofílico. 


\section{AGRADECIMENTOS}

À FAPESB pela bolsa de mestrado concedida e a CAPES pelo apoio financeiro ao Projeto.

\section{BIBLIOGRAFIAS}

CHEN, W., YAN, L., BANGAL, P. R., "Preparation of graphene by the rapid and mild thermal reduction of graphene oxide induced by microwaves", Carbon, v. 48, pp. II46-II52, 2010. Disponível em: DOI: Io.1or6/j.carbon.2009.11.037

FRACKOWIAK, E., BÉGUIM, F., “ Review Carbon materials for the electrochemical storage of energy in capacitors, Carbon, v. 39, pp. 937-950, 2001. Disponível em: DOI:10.1016/Sooo8-6223(oo)oor83-4

FERRARI, A. C., "Raman spectroscopy of graphene and graphite: Disorder, electron-phonon coupling, doping and nonadiabatic effects", Solid State Communications, v. I43, n. I-2, pp. 47-57, 2007. Disponível em: DOI: 10.1016/j.ssc.2007.03.052

FERRARI, A. C., MEYER, J. C. SCARDACI, V., CASIRAGHI, C., LAZZERI, M., MAURI, F., PISCANEC, S., JIANG, D., NOVOSELOV, K. S., ROTH, S., GEIM, A. K., "Raman Spectrum of Graphene and Graphene Layers", Physical Review Letters, v. 97, n. I8, pp. I87401, 2006. Disponível em: DOI: 10.1103/PhysRevLett.97.187401

GEIM, A., NOVOSELOV,K., For groundbreaking experiments regarding the twodimensional material graphene, The Nobel Prize in Physics, 2010. Disponível em: https://www.nobelprize.org/prizes/physics/2010/press-release/ . Acesso em 26/o7/2021

GIRALDO, A. V., "El Grafeno", Revista Colombiana de Materiales, v. I, n. 53, pp. o-24, 2011. Disponível em: https://revistas.udea.edu.co/index.php/materiales/article/view/9172. Acesso em 26/07/2021

HUMMERS, W. S., HOFFMANN, R. E., "Preparation of Graphitic Oxide", v. 208, n. 1937, pp. 1937, 1957. Disponível em: DOI: 10.102I/jaor539aor7

IIJIMA, S., Helical microtubules of graphitic carbon, Nature, v. 354, pp. 56-58, Nov. I99I. Disponível em: DOI: 10.1038/354056ao

JIN, Y., HUANG, S., ZHANG, M., JIA, M., HU, D., "A green and efficient method to produce graphene for electrochemical capacitors from graphene oxide using sodium carbonate as a reducing agent", Applied Surface Science, v. 268, pp. 541-546, 2013. Disponível em: DOI:10.1016/j.apsusc.2013.01.004

KIM, K. S., ZHAO, Y., JANG, H., LEE, S. Y., KIM, J. M., KIM, K. S., et al., "Large-scale pattern growth of graphene films for stretchable transparent electrodes", Nature, v. 457, n. 7230, pp. 706-710, 2009. Disponível em: DOI: 10.1038/natureo7719 
LIU, Panbo; HUANG, Ying; WANG, Lei., "A facile synthesis of reduced graphene oxide with $\mathrm{Zn}$ powder under acidic condition", Materials Letters, v. 91, pp. 125-128, 2013. Disponível em: DOI: 10.1016/j.matlet.2012.09.085.

LIAO K. H., MITTAL A., Bose S., Leighton C., Mkhoyan K. A, Macosko C. W., "Aqueous only route toward graphene from graphite oxide", ACS Nano, v. 5, n. 2, pp. 1253-1258, 2011. Disponível em: DOI: I0.102I/nnio28967

LOH, K. P., BAO, Q., ANG, P. K., YANG, J.,“The chemistry of grapheme”, Journal of Materials Chemistry, v. 20, n. 12, pp. 2277-2289, 2010. Disponível em: DOI: 10.1039/B920539J

NOVOSELOV AK., GEIM A., et al.,"Electric field effect in atomically thin carbon films”, Science, v. 306, n. 5696, pp. 666-669, 2004. Disponível em: DOI: Io.II26/science.1102896

PEI, S., CHENG, H. M., "The reduction of graphene oxide", Carbon, v. 5o, n. 9, pp. 32103228, 2012. Disponível em: DOI: Io.IoI6/j.carbon.20II.II.oIo

RUMMELI, M. H., BACHMATIUK, A., SCOTT, A., BORRNERT, F., WARNER, J.H., Hoffmann, V., Lin, J-H., Cuniberti, G., Buchner, B., "Direct low-temperature nanographene CVD synthesis over a dielectric insulator", ACS nano, v. 4, n. 7, pp. 4206-4210, 2010. Disponível em DOI: 10.102I/nnioo97is

STANKOVICH, S., DIKIN, D. A., PINER, R. D., KOHLHAAS, K. A., KLEINHAMMER, A., JIA, Y., et al., "Synthesis of graphene-based nanosheets via chemical reduction of exfoliated graphite oxide", Carbon, v. 45, n. 7, pp. 1558-1565, 2007. Disponível em: DOI: I0.1016/j.carbon.2007.02.034

TUINSTRA, F. KOENIG, J. L., "Raman spectrum of graphite", The Journal of Chemical Physics, v. 53, n. 3, pp. I126-1130, 1970. Disponível em DOI: 10.1016/j.matlet.2012.09.085

WANG, Y. Y.,Ni, Z. H., Yu, T., Shen, X., Wang, H. M., Wu, Y. H., Chen, W., Wee, A.T.S.,"Raman studies of monolayer graphene: The substrate effect, Journal of Physical Chemistry C, v. II2, n. 29, pp. 10637-10640, 2008. Disponível em: DOI: I0.1021/jp8008404

YUE Z, LIQIANG R, SHIREN W, "Functionalization of graphene sheets through fullerene attachment", Journal of Materials Chemistry, n. 21.pp. 5386, 2011. DOI: 10.1039/CIJMio257E.

ZHU W, STOLLER M. D., CAI W., et al., "Exfoliation of graphite oxide in propylene carbonate and thermal reduction of the resulting graphene oxide platelets", ACS Nano, v. 4, n. 2 pp. 1227-I233, 2010.Disponível em: DOI: 10.1021/nn9or689k 